VoL. 48 (1993) [47-53]

\title{
ON THE WIDTH OF A PLANAR CONVEX SET CONTAINING ZERO OR ONE LATTICE POINTS
}

PAUL R. SCOTT

\begin{abstract}
We generalise to a rectangular lattice a known result about the maximal width of a planar compact convex set containing no points of the integral lattice. As a corollary we give a new short proof that the planar compact convex set of greatest width which contains just one point of the triangular lattice is an equilateral triangle.
\end{abstract}

\section{INTRODUCTION}

In the Euclidean plane $E^{2}$ let $\Lambda$ denote the integral lattice, and $\Lambda_{T}$ the triangular lattice generated by the vectors $(1,0)$ and $(1 / 2, \sqrt{3} / 2)$. Let also $\Lambda_{R}(u, v)$ denote the rectangular lattice generated by the vectors $(u, 0)$ and $(0, v)$.

We denote by $K$ a compact, convex set in $E^{2}$. The minimal width, $w(K)$, of $K$ is the smallest distance between parallel supporting lines of $K$. A number of results are known about $w(K)$ in the case where $K$ is constrained by a lattice:

THEOREM 1. If $K$ contains no points of the lattice $\Lambda$ in its interior, then $w(K) \leqslant$ $(\sqrt{3}+2) / 2$; equality is assumed for an equilateral triangle of side-length $(\sqrt{3}+2) / \sqrt{3}$ [2].

THEOREM 2. If $K$ contains one point of the lattice $\Lambda$ in its interior, then $w(K) \leqslant$ $\sqrt{2}+1$; equality is assumed here for a certain isosceles triangle [3].

THEOREM 3. If $K$ contains no points of the lattice $\Lambda_{T}$ in its interior, then $w(K) \leqslant \sqrt{3}$; equality is assumed here for the 'obvious' equilateral triangle of sidelength 2 [4].

Recently a further result of this type has been proved [5]:

THEOREM 4. If $K$ contains one point of the lattice $\Lambda_{T}$ in its interior, then $w(K) \leqslant 3 / 2 \sqrt{3}$; equality is assumed here for an equilateral triangle of side-length 3 . (See Figure 1(a).)

The proof given for this pretty result is long, but can be halved in length by building on known results. Here we shall prove the following simple generalisation of Theorem 1 , from which Theorem 4 will immediately follow as a corollary.

Received 22nd July, 1992

Copyright Clearance Centre, Inc. Serial-fee code: 0004-9729/93 \$A2.00+0.00. 
THEOREM 5. Suppose that $u \leqslant v$ and $v \leqslant \sqrt{3} u$. If $K$ contains no point of $\Lambda_{R}(u, v)$ in its interior, then $w(K) \leqslant(\sqrt{3} u+2 v) / 2$. This result is best possible, equality being attained for the equilateral triangle illustrated in Figure $1(b)$.

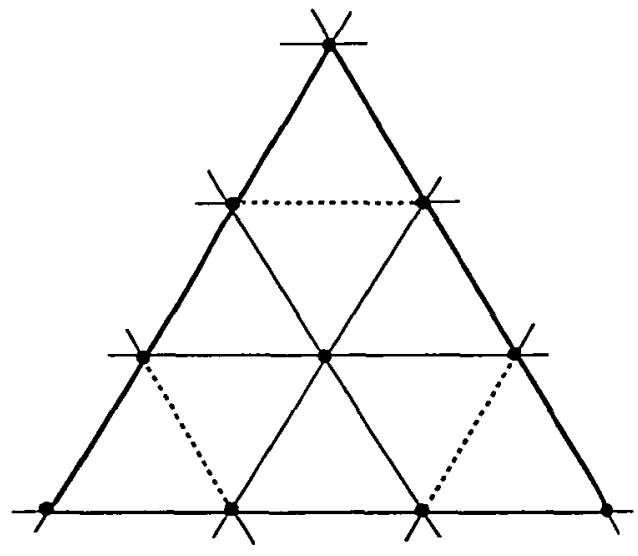

(a)

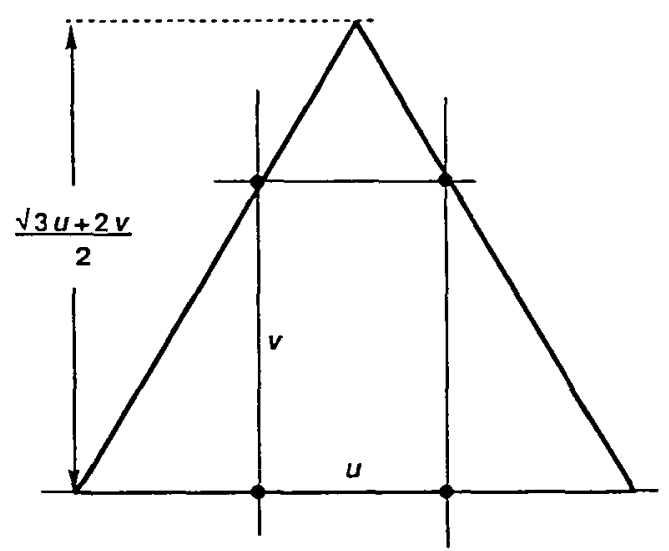

(b)

Figure 1.

\section{ProOF of TheOREM 5}

To prove Theorem 5, we shall need to step through the proof of Theorem 1 given in [2], to which we refer the reader. In replacing $\Lambda$ by $\Lambda_{R}(u, v)$, several significant modifications are required.

We shall assume that $K$ is a planar compact convex set satisfying the conditions of Theorem 5, and for which $w(K)$ is as large as possible. From Figure 1(b) we see that $w(K) \nless(\sqrt{3} u+2 v) / 2$. We may therefore use the well-known result of Blaschke [1] and suppose that $K$ contains a disc of radius

$$
R=\frac{1}{3} \cdot \frac{1}{2} \cdot(\sqrt{3} u+2 v)
$$

By translating $K$ through a suitable lattice vector, we can take the centre of this disk to lie inside the rectangle with vertices $A(0,0), B(u, 0), C(u, v), D(0, v)$. Now from the bounds on $u$ and $v, R \geqslant(\sqrt{3}+2) u / 6>u / 2$, and $R \geqslant v / 2$. It follows that the disc must intercept (at least) three sides of the rectangle $A B C D$. We can now argue as in [2] to deduce that $K$ lies within a convex quadrilateral $Q$ determined by lines $a, b, c, d$ through $A, B, C, D$ respectively. Since $w(K) \leqslant w(Q)$, and $Q$ contains no lattice points in its interior, it is sufficient to show that $w(Q) \leqslant(\sqrt{3} u+2 v) / 2$.

In quadrilateral $Q$, let $a . b=L, b . c=M, c . d=N, d . a=P$. No pair of sides of 
$Q$ can be parallel. For if say sides $a$ and $c$ are parallel, then

$$
w(Q) \leqslant A C=\sqrt{u^{2}+v^{2}} \leqslant \sqrt{2} v \leqslant v+(\sqrt{2}-1) \sqrt{3} u=v+\frac{\sqrt{3} u}{\sqrt{2}+1} \leqslant(2 v+\sqrt{3} u) / 2 .
$$

Hence we may think of $Q$ as the intersection of two triangles as in Figure 2. Let $L$ be the vertex which these two triangles have in common. Then the diagonal $M P$ will be called the minor diagonal of $Q$. (There are in fact two cases to consider here when $R$ is not a square: if $P$ say is the vertex in common, we ultimately obtain a different result. For the present we shall stay with the notation of Figure 2, and evaluate the two cases at the end of the argument.)

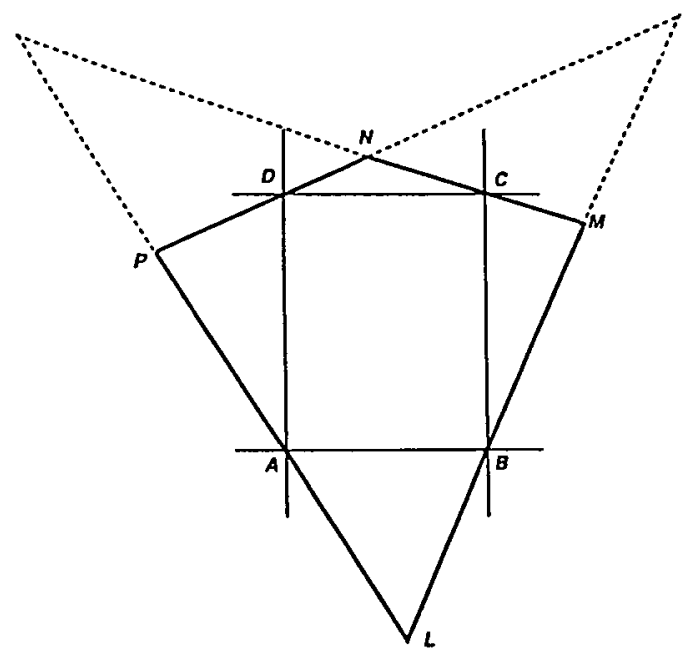

Figure 2.

Following [2], we now use Steiner symmetrisation. If $K$ is a given region and $l$ a given line in the plane, we obtain a new region $K^{\prime}$ as follows. Displace each chord $X Y$ of $K$ which is perpendicular to $l$ along the line $X Y$ until its midpoint lies on $l$. Then $K^{\prime}$ is the union of these displaced chords.

The proof of the theorem now follows from four simple lemmas. The first lemma is unchanged from [2].

LEMмa 1. Let $Q^{\prime}$ be the kite obtained from quadrilateral $Q$ under symmetrisation about the perpendicular bisector of the minor diagonal of $Q$. Then $w\left(Q^{\prime}\right) \geqslant w(Q)$.

Lemma 2. Let $m$ denote the length of the minor diagonal of $Q$, and let $n$ denote the width of $Q$ in a direction perpendicular to this. Let $R_{\lambda}$ be a rectangle obtained from $R$ under a similarity with scale factor $\lambda$. If $R_{\lambda}$ is inscribed in $Q$ with a vertex 
on each side of $Q$, then

$$
\lambda \geqslant \frac{m n}{v m+u n} .
$$

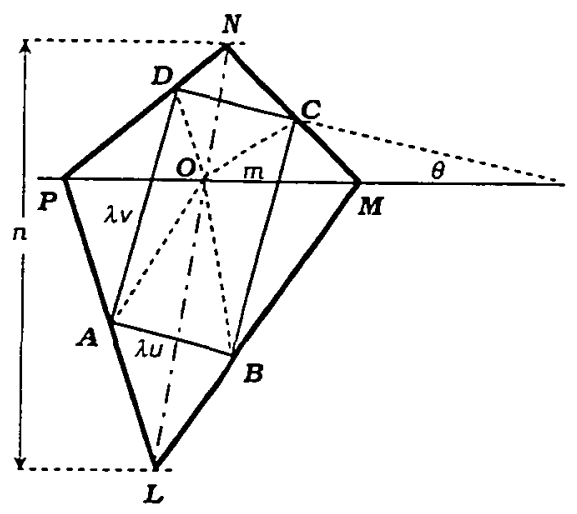

Figure 3.

Proof: Let $R_{\lambda}$ have vertices $A, B, C, D$ and let $D C$ make an angle $\theta$ with the minor diagonal as in Figure 3. Let the diagonals of $Q$ meet in the point $O$.

Now the area of $Q$ is $m n / 2$. This area is also given by adding the areas of quadrilaterals $O D P A, O B M C$ to the areas of $O C N D, O A L B$. We obtain

$$
\lambda v \cos \theta . m / 2+\lambda u \cos \theta . n / 2=m n / 2 .
$$

Hence

$$
\begin{gathered}
\lambda(v m+u n) \cos \theta=m n, \\
\lambda(v m+u n) \geqslant m n, \\
\lambda \geqslant \frac{m n}{v m+u n}
\end{gathered}
$$

or

as required.

We observe that equality holds when the rectangle has a pair of sides parallel to the minor diagonal.

The third lemma requires only minor alteration from that given in [2].

IEMma 3. The quadrilateral $Q$ can be transformed into a kite $Q^{\prime}$ having the following properties:

(a) $w\left(Q^{\prime}\right) \geqslant w(Q)$;

(b) $Q^{\prime}$ contains no lattice point in its interior;

(c) $Q^{\prime}$ has its axis along the line $x=a / 2$;

(d) the sides of $Q^{\prime}$ pass through $A, B, C, D$ respectively. 
Proof: Transform the quadrilateral $Q$ as in Lemma 1 to obtain a kite $Q^{\prime}$ with $\boldsymbol{w}\left(Q^{\prime}\right) \geqslant \boldsymbol{w}(Q)$.

Since $Q$ and $R$ ( $=R_{1}$ ) exhibit the relationship outlined in Lemma 2, the constants $m$ and $n$ satisfy the condition $m n /(v m+u n) \leqslant 1$. Now inscribe a rectangle $R_{\lambda}$ similar to $R$ in the kite $Q^{\prime}$, with sides parallel to the diagonals of $Q^{\prime}$. Since $m$ and $n$ are invariant under the symmetrisation, and the sides of $R_{\lambda}$ are parallel to the diagonals of $Q^{\prime}$, we obtain $\lambda=m n /(v m+u n) \leqslant 1$. If $\lambda=1$, we take $R_{\lambda}$ to coincide with $R$, and $Q^{\prime}$ now satisfies the conditions of the lemma. If $\lambda<1$, an enlargement of $Q^{\prime}$ (and $R_{\lambda}$ ) with scale factor $1 / \lambda(>1)$ increases its minimal width, and gives the required kite.

LEMмA 4. $w\left(Q^{\prime}\right) \leqslant(\sqrt{3} u+2 v) / 2$, with equality occurring only when $Q^{\prime}$ degenerates into an equilateral triangle.

Proof: Using the obvious labelling corresponding to that of $Q$, let $Q^{\prime}=L^{\prime} M^{\prime} N^{\prime} P^{\prime}$ be the kite for which $w\left(Q^{\prime}\right)$ is maximal. Then exactly as in [2], we deduce that the four altitudes of $Q^{\prime}$ must be equal, and that the angles at $L^{\prime}, M^{\prime}$ and $P^{\prime}$ are congruent.

Consider now the half-kite illustrated in Figure 4.

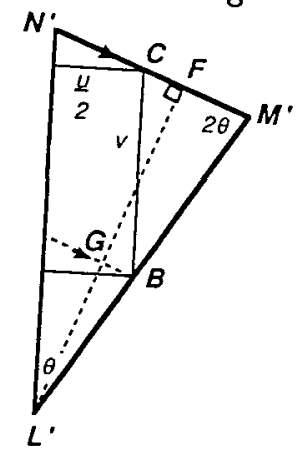

Figure 4.

Let the width of $Q^{\prime}$ be denoted by $p(\theta)$. Then

$$
\begin{aligned}
p(\theta)=F L^{\prime} & =F G+G L^{\prime} \\
& =v \sin 3 \theta+(u \operatorname{cosec} \theta \sin 2 \theta) / 2 \\
& =v \sin 3 \theta-u \cos \theta \\
p^{\prime}(\theta) & =3 v \cos 3 \theta-u \sin \theta \\
& <0
\end{aligned}
$$

hence

for all (positive) values of $u$ and $v$, and for the allowable range of $\theta$

$$
\frac{\pi}{6} \leqslant \theta<\frac{\pi}{3}
$$

Hence the maximal value of $p$ is assumed for $\theta=\pi / 6$, and $Q^{\prime}$ is an equilateral triangle. 
We have now shown that if $Q^{\prime}$ is a quadrilateral with maximal width, and such that it is derived from a quadrilateral $Q$ determined by two intersecting triangles with common vertex $L$ (see Figure 2), then $w\left(Q^{\prime}\right) \leqslant p_{\max }=(\sqrt{3} u+2 v) / 2$. As mentioned earlier, there is another possibility, where $P$ (say) rather than $L$, is taken as the common vertex of the defining triangles. In this case the rôles of $u$ and $v$ are interchanged, and the maximal value for the width is given by $p^{*}=(\sqrt{3} v+2 u) / 2$. However it is easily checked that $\boldsymbol{p}^{*}<\boldsymbol{p}_{\max }$ for $u<v$.

To complete the proof of the theorem, we only need to observe that no closed, convex, proper subset of the equilateral triangle has the same minimal width as the triangle. For no such subset can contain all three vertices of the triangle, and removal of any vertex of the triangle decreases the width.

\section{Proof of ThEOREM 4}

The extremal equilateral triangle in Figure 1(a) contains an inscribed regular hexagon of side-length 1 . The endpoints of the two horizontal edges of this hexagon determine a rectangle $R$, which in turn determines a rectangular lattice $\Lambda_{R}(u, v)$ for which $u=1$ and $v=\sqrt{3}$. The convex set $K$ which satisfies the conditions of Theorem 5 can contain no points of this lattice. Hence, from Theorem 5,

$$
w(K) \leqslant(\sqrt{3} u+2 v) / 2=(\sqrt{3}+2 \sqrt{3}) / 2=3 \sqrt{3} / 2,
$$

and equality occurs here if and only if $\mathrm{K}$ is the equilateral triangle of side-length 3.

This completes the proof of Theorem 4.

\section{Some final Comments}

There are two comments which should be made. The first is that the condition $v \leqslant \sqrt{3} u$ in the statement of Theorem 5 may not be necessary. However, including this constraint helps keep the proof simple, and is sufficient to establish Theorem 4, which is our main goal. It is interesting that equality occurs precisely for the values of $u$ and $v$ which give the chosen sublattice of the triangular lattice.

The second comment concerns the nature of the proof. Although the proof given here is much shorter than that given in [5], it is still not entirely satisfying. The result is 'obvious' in the sense that the extremal equilateral triangle fits naturally with the structure of the triangular lattice. One might have hoped that symmetry considerations would have led to an immediate proof, as indeed happened in the proof of Theorem 3. Perhaps there is an even shorter proof remaining to be discovered! 


\section{REFERENCES}

[1] W. Blaschke, Kreis und Kugel (W. de Gruyter, Berlin, 1956).

[2] P.R. Scott, 'A lattice problem in the plane', Mathematika 20 (1973), 247-252.

[3] P.R. Scott, 'On planar convex sets containing one lattice point', Quart. J. Math. Oxford Ser. 236 (1985), 105-111.

[4] P.R. Scott, 'Convex sets and the hexagonal lattice', Math. Mag. 51 (1978), 237-238.

[5] K. Wetwitschka, 'Zur Breite konvexer Mengen mit einem inneren Gitterpunkt im triangularen Gitter', Ann. Univ. Sci. Budapest 34 (1991), 121-135.

Department of Mathematics The University of Adelaide Adelaide SA 5001

Australia 